\title{
Minimal Length Requirement of the Single-stranded Tails for Ligation-independent Cloning (LIC) of PCR Products
}

\author{
Charalampos Aslanidis, ${ }^{1}$ Pieter J. de Jong, ${ }^{2}$ and Gerd Schmitz ${ }^{1}$
}

${ }^{1}$ Institute for Clinical Chemistry, University of Regensburg, 93053 Regensburg, Germany; ${ }^{2}$ Human Genetics Department, Roswell Park Cancer Institute, Buffalo, New York 14263

The ligation-independent cloning of PCR products (LIC-PCR) is a versatile and highly efficient cloning procedure resulting in recombinant clones only. Recombinants are generated between PCR products and a PCRamplified vector through defined complementary single-stranded (ss) ends artificially generated with T4 DNA polymerase. This procedure does not require restriction enzymes, alkaline phosphatase, or DNA ligase. The primers used for amplification contain an additional 12-nucleotide sequence at their 5 'ends that is complementary in the vector- and insertspecific primers. The $\left(3^{\prime} \rightarrow 5^{\prime}\right)$ exonuclease activity of T4 DNA polymerase is used in combination with a predetermined dNTP (dGTP for the inserts and dCTP for the vector) to specifically remove 12 nucleotides from each 3 'end of the PCR fragments. Because of the complementarity of the ends that are generated, circularization can occur between vector and insert. The recombinant molecules do not require in vitro ligation for efficient bacterial transformation. To make this technique widely applicable, we have simplified the handling of the PCR fragments prior to LIC. The PCR products do not need further purification following the T4 DNA polymerase treatment. Incubation of vector and insert PCR fragments for as little as $\mathbf{5}$ min is sufficient for a high yield of recombinants. Comparison of the transformation efficiencies using different- length LIC tails revealed that using 12-nucleotide cohesive ends produced four times more transformants than were obtained with the LIC with 10-nucleotide cohesive ends. When the LIC tails were 8 nucleotides long, no transformants were obtained. PCR fragment purification, T4 DNA polymerase treatment, and LIC is complete in $<1 \mathrm{hr}$.

E or many applications, $\mathrm{PCR}^{(1)}$ has replaced molecular cloning as the method of choice for the rapid amplification and isolation of specific DNA sequences from genomic DNA. Nevertheless, complex PCR product mixtures still require cloning for the isolation of specific products, for example, for use as probes. This is the case for PCR libraries established from microdissected chromosomes ${ }^{(2,3)}$ and for inter-Alu PCR. ${ }^{(4-6)}$ In some cases, specifically generated PCR products from cDNAs of interest have to be cloned in specific vectors for expression studies. To facilitate cloning, restriction endonuclease sites may be introduced into the amplification primers so that subsequent digestion of the PCR fragments with the appropriate enzymes results in products ready to be cloned in specific sites of vectors. Depending on the recognition site used in the primers, the cleavage may be more or less efficient. Some restriction endonucleases require additional nucleotides at their 5'end. Blunt end cloning is less efficient and requires the removal of the $3^{\prime}$ overhang gener- ated by Taq polymerase. ${ }^{(7,8)}$ In both cases, it is necessary to prevent high nonrecombinant backgrounds by treating the vector with alkaline phosphatase. Despite this treatment, a considerable fraction of the clones will lack inserts. Recently, several alternative methods have been developed to circumvent these problems. These methods can be divided in the ligation-dependent and -independent approaches.

In 1991, we were the first group to develop a highly efficient ligation-independent cloning procedure for PCR fragments (LIC-PCR) that does not involve restriction enzymes, alkaline phosphatase, T4 polynucleotide kinase, or DNA ligase. ${ }^{(9)}$ In essence, 12-nucleotidelong single-stranded (ss) tails are created at the ends of the PCR products and the PCR-amplified plasmid vector using T4 DNA polymerase. The ss ends present at the PCR fragments are complementary to those attached to the vector. Noncovalent associations between the vector and PCR fragments are facilitated. The vector has identical, noncomplementary tails at either end, preventing the formation of circular forms consisting of vector only. These "recombinant" forms are used to transform Escherichia coli very efficiently. We have not detected any nonrecombinant transformants since then. The procedure seems to result in $100 \%$ recombinant clones. Another LIC procedure was developed by Shuldiner et al. ${ }^{(10,11)}$ This method makes use of denaturation and heterologous annealing of the PCR product and the vector and is difficult to control. Rasht- 
chian et al. ${ }^{(12)}$ have developed a LIC procedure that is very similar to ours. The method is based on the addition of a 12base dUMP-containing sequence to the 5 'end of PCR primers. Selective degradation of the dUMP residues in the PCR products with uracil DNA glycosylase (UDG) generates the required 12-nucleotide-long ss tails.

Other ligation-dependent cloning procedures using T4 DNA polymerase to generate smaller ss tails have been developed. ${ }^{(13,14)}$ In these cases, the vector is not amplified but is cut by specific restriction endonucleases to generate the compatible ends for cloning. Because the ss tails are small, a ligation step is necessary prior to transformation. Another LIC procedure makes use of the specific addition of 1 nucleotide at the 3 'end of the PCR product by Taq polymerase. Vectors have been constructed from various groups that contain the complementary nucleotide at the ends from the linearized vector to facilitate the cloning. ${ }^{(15-17)}$ These so called T-vectors however, are confined to the use of Taq polymerase. Other thermostable DNA polymerases, like Pfu polymerase, which lack the terminal transferase activity, cannot be used. ${ }^{(18)}$

Based on our LIC procedure, Haun and Moss have constructed a plasmid vector that allows the LIC of CDNAs in any reading frame and directs their synthesis in E. coli as glutathione S-transferase (GST)-linked fusion proteins. ${ }^{(19)}$ They confirm that the procedure is very simple, rapid, and highly efficient. In contrast with our protocol they use a plasmid vector that, upon digestion with NarI, allows the generation of the 12-nucleotide ss tails by T4 DNA polymerase. ${ }^{(20)}$ The LIC procedure has been used by others for cloning and expression of various cDNAs. ${ }^{(21,22)}$ Now, commercially available cloning kits adapted from LIC are available. More recently, sophisticated expression vectors to be used in mammalian or baculoviral systems have been constructed by S. Gruenwald and S. Singh (PharMingen, San Diego).

The requirement for an additional 12 nucleotides at the 5 'end of the PCR primers may prevent people from considering this technique as the method of choice. To keep the costs for the primers low and make this procedure widely applicable, here we test the hypothesis that ss tails shorter than 12 bases long permit efficient LIC of PCR products. We have compared the cloning efficiencies of Alu PCR products having 12-, 10-, and 8-nucleotide-long ss tails. Furthermore, we have simplified the purification steps required for LIC. PCR fragment purification, T4 DNA polymerase treatment, and LIC is complete in $<1 \mathrm{hr}$.

\section{MATERIALS AND METHODS \\ Enzymes, Reagents, and Oligonucleotides}

Taq polymerase and buffer were from Perkin-Elmer Cetus; T4 DNA polymerase and buffer were from Boehringer Mannheim. Competent bacteria (Max Efficiency DH5 $\alpha$ ), the 1-kb ladder, and the 123-bp ladder were purchased from BRL. Oligonucleotides were synthesized and HPLC-purified by MWG (Ebersberg, Germany). The DNA sequences of the individual primers are as follows: PDJ8312, 5' -gatggtagtaggCCACTGCACTCCAGCC-3'; PDJ8310, 5' -tggtagtaggCCAC'TGCACTCCAGCC-3'; PDJ8308, 5' -gtagtaggCCACTGCACTCCAGCC-3'; PDJ8012, 5'-cctactaccatcGGATCCCCGGGT-3'; PDJ8010, 5'-cctactaccaGGATCCCCGGGT3'; PDJ8008, 5'-cctactacGGATCCCCGGGT-3'; PDJ8112, 5'-cctactaccatcGTCGACCTGCAG-3'; PDJ8110, 5'-cctactaccaGTCGACCTGCAG-3'; PDJ8108， 5'-cctactacGTCGACCTGCAG- $3^{\prime}$. The primers with the 12-nucleotide tails are identical to PDJ81, PDJ82, and PDJ83. ${ }^{(9)}$

\section{Amplification of Vector and Human DNA}

All PCR reactions were performed in 50$\mu l$ mixtures using the following buffer conditions: $50 \mathrm{~mm} \mathrm{KCl}, 10 \mathrm{~mm}$ Tris- $\mathrm{HCl}$ (pH 8.3), $1.5 \mathrm{~mm} \mathrm{MgCl}_{2}, 0.2 \mathrm{~mm}$ of each dNTP, and $0.5 \mu \mathrm{M}$ each oligonucleotide. Amplification was for 35 cycles in a Perkin-Elmer Cetus thermal cycler (9600) as follows: initial heating to $94^{\circ} \mathrm{C}$ for $5 \mathrm{~min}$; then $44 \mathrm{sec}$ at $92.3^{\circ} \mathrm{C}, 40 \mathrm{sec}$ at $60.8^{\circ} \mathrm{C}$, and $46 \mathrm{sec}$ at $71.5^{\circ} \mathrm{C}$ for 35 cycles; and a final extension at $72^{\circ} \mathrm{C}$ for $5 \mathrm{~min}$ prior to cooling to $4^{\circ} \mathrm{C}$. The vector was amplified starting with $1 \mathrm{ng}$ of $\mathrm{Xbal}$-linearized pUC19 plasmid, ${ }^{(23)} 25$ pM of each PCR primer, and 2 units of Taq polymerase. Prior to PCR, pUC19 was cut with $\mathrm{XbaI}$ as follows: $100 \mathrm{ng}$ of pUC19 was incubated with 15 units of $\mathrm{XbaI}$ (overkill) at $37^{\circ} \mathrm{C}$ for 30 min in a $20-\mu$ l volume. $\mathrm{H}_{2} \mathrm{O}$ was added to a final volume of $100 \mu \mathrm{l}$, the reaction denatured at $95^{\circ} \mathrm{C}$ for $10 \mathrm{~min}$, and chilled on ice. Inter-Alu sequences were amplified using 25 pM of the appropriate Alu primer, $100 \mathrm{ng}$ of human genomic DNA, and 2 units of Taq polymerase. The same cycling program was used for the inter-Alu PCR as described for the vector PCR. Products from PCR reactions were usually analyzed by electrophoresis in 2-3\% agarose gels (NuSieve GTG) in TBE buffer with ethidium bromide. ${ }^{(24)}$ One $50-\mu l$ PCR reaction resulted in $2-3 \mu \mathrm{g}$ of linearized pUC19.

\section{T4 DNA Polymerase Treatment}

The amplified vector and inter-Alu PCR products were purified using a glass powder suspension (Gene-CleanII from BIO101, San Diego) as recommended by the supplier. For the generation of the ss tails, the purified DNA preparations were treated with 2 units T4 DNA polymerase in a $50-\mu$ l volume $[50 \mathrm{~mm}$ Tris- $\mathrm{HCl}(\mathrm{pH}$ $8.8), 15 \mathrm{~mm}$ ammonium sulfate, $7 \mathrm{~mm}$ magnesium chloride, $0.1 \mathrm{~mm}$ EDTA, 10 $\mathrm{mm}$ mercaptoethanol, and $200 \mu \mathrm{g} / \mathrm{ml}$ of bovine serum albumin] in the presence of dGTP $(0.5 \mathrm{~mm})$ for the Alu-PCR products or dCTP $(0.5 \mathrm{~mm})$ for the vector. After incubation for $20 \mathrm{~min}$ at $37^{\circ} \mathrm{C}$, the mixtures were heated for $10 \mathrm{~min}$ at $65^{\circ} \mathrm{C}$. Prior to LIC, the Alu-PCR products and pUC19 vector were either purified using the GeneClean procedure or were used without further purification. The vector was diluted in TE buffer to $5 \mathrm{ng} / \mu \mathrm{l}$ and the Alu PCR products were diluted to 10 $20 \mathrm{ng} / \mu \mathrm{l}$ (in TE buffer).

\section{Cloning and Transformation}

A 2- $\mu 1$ aliquot of T4 DNA polymerasetreated pUC19 (10 ng) was combined with $4 \mu \mathrm{l}$ of T4 DNA polymerase-treated Alu PCR products $(60 \mathrm{ng})$ in a $20-\mu \mathrm{l}$ volume (TE buffer). After a 1-hr incubation at room temperature ( 5 min works just as well), $5 \mu \mathrm{l}$ was used to transform $50 \mu \mathrm{l}$ of competent cells (in 1.5-ml Eppendorf micro test tubes) with BRL-recommended procedures. The temperature for the heat shock of the cells for DNA uptake is crucial to the experiment and was monitored carefully (precisely $\left.42^{\circ} \mathrm{C}\right) . \operatorname{SOC}^{(24)}$ medium $(0.45 \mathrm{ml})$ was added, and the cells were incubated for one hour at $37^{\circ} \mathrm{C}$ in Eppendorf thermomixer 5436 at $1000 \mathrm{rpm}$. From the bacterial suspension, $100 \mu \mathrm{l}$ and $400 \mu \mathrm{l}$ (upon concentration by centrifugation) 
were plated on LB agar plates ${ }^{(24)}$ supplemented with $100 \mu \mathrm{g} / \mathrm{ml}$ of ampicillin.

\section{PCR of Transformants and DNA Sequencing}

Transformants were checked for the presence of recombinant plasmids by PCR using the universal and the reverse primers flanking the multicloning site in pUC19. Bacterial colonies were transferred into the PCR mixture just by touching the colony using disposable pipette tips and pipetting up and down in the PCR mixture. The buffer conditions, primer concentrations, and cycling program were as mentioned above. Aliquots $(5 \mu \mathrm{l})$ were analyzed by agarose gel electrophoresis. The DNA sequencing of the clones was done on the Pharmacia ALF sequencer using fluorescence-labeled universe and reverse pUC19 primers.

\section{RESULTS \\ Principle of LIC}

The LIC of PCR products is outlined in Figure 1. It is based on the use of short cohesive ends (8-12 nucleotides) at the clonable PCR products as well as the vector. The pUC19 plasmid vector is amplified using two primers complementary to the multicloning site. The vector primers PDJ8012 and PDJ8112 contain 12 additional nucleotides at their $5^{\prime}$ end lacking dGMP residues. As a consequence, the 12 nucleotides at the $3^{\prime}$ ends of the amplified vector lack dCMP. In the presence of T4 DNA polymerase and dCTP, the 3' ends are degraded by the $3^{\prime} \rightarrow 5^{\prime}$ exonuclease activity of the polymerase. Because of the primer design, the exonuclease stops at nucleotide 13, which is dCMP.

Clonable Alu-PCR fragments were generated using Alu primer PDJ8312 and human genomic DNA as a template. In addition to its 17-nucleotide Alu repeatspecific sequence, ${ }^{(25-27)}$ this primer contains at its 5 ' end the complementary 12 nucleotides from the pUC19 primers. Consequently, ss tails can be generated using T4 DNA polymerase and dGTP.

\section{Cloning of PCR Products}

Since the publication of $\mathrm{LIC}^{(9)}$ we have used this procedure to clone numerous PCR fragments. It is noteworthy that in the course of the last 4 years, all of the

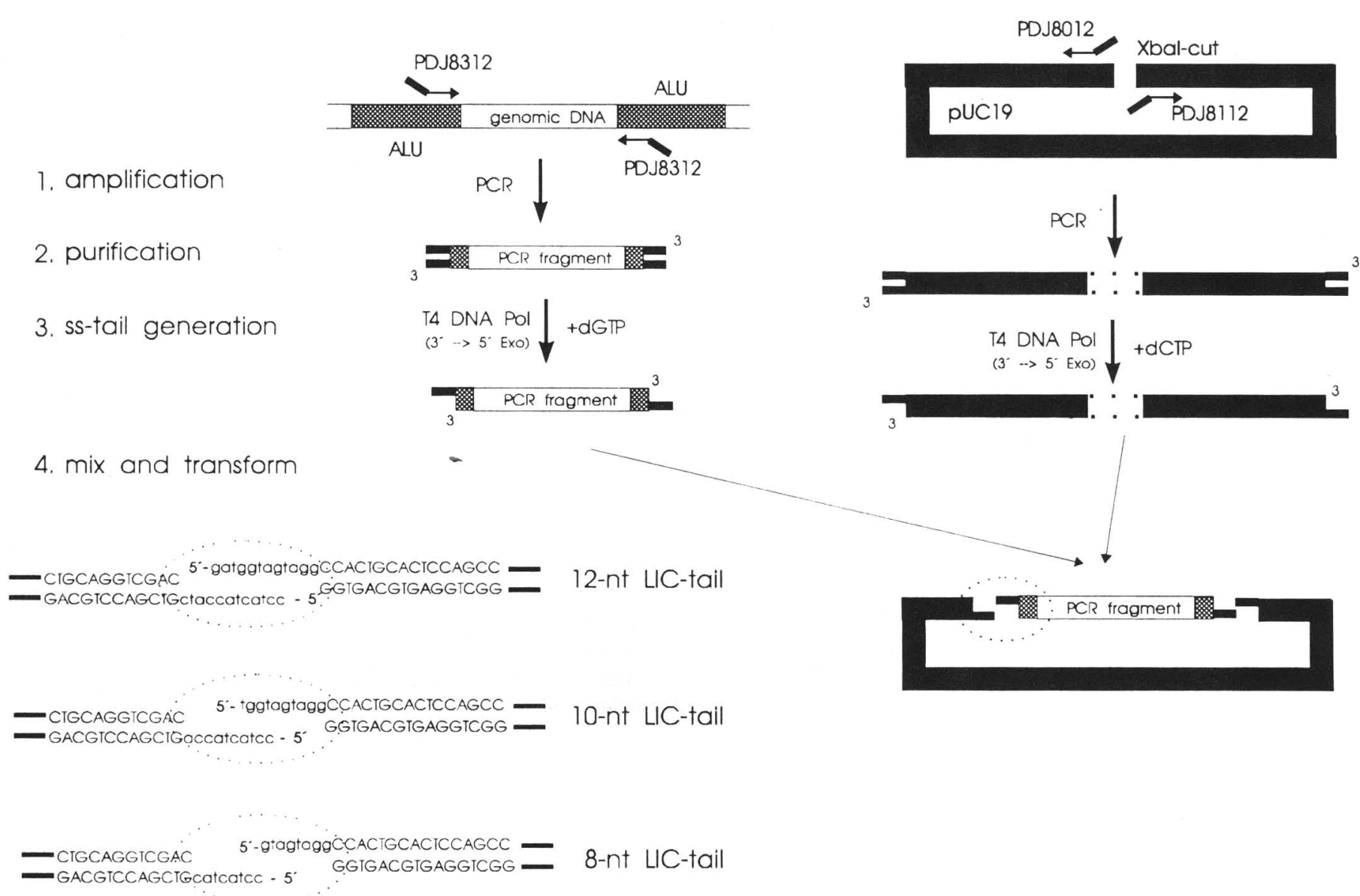

FIGURE 1 Generation of defined ss ends on PCR products. Inter-Alu repeat sequences are amplified using one of the three Alu primers (PDJ8312, PDJ8310, and PDJ8308). These primers are partly homologous to the consensus Alu sequence ${ }^{(27)}$ and contain either 12 , 10, or 8 additional nucleotides at their $5^{\prime}$ ends that are required to generate ss tails in the PCR products. XbaI-cut pUC19 is amplified with the primer pairs PDJ8012/ PDJ8112, PDJ8010/PDJ8110, or PDJ8008/PDJ8108 that are partly homologous to the multicloning site and contain 12,10 , or 8 additional nucleotides at their $5^{\prime}$ ends. The PCR products are digested with the $\left(3^{\prime} \rightarrow 5^{\prime}\right)$ exonuclease associated with T4 DNA polymerase in the presence of dGTP (inter-Alu fragments) or dCTP (pUC19) to generate 12-, 10-, or 8-nucleotide-long ss ends. The $5^{\prime}$ overhanging ends from the Alu PCR products and vector are complementary (lower left corner) and allow recombinant molecules to be formed without using DNA ligase. 
randomly picked transformants from different experiments proved to contain inserts (several hundred analyzed). We have simplified the cloning procedure in regard to the incubation buffer, incubation times, and purification of the PCR fragments. In this study we compare LIC efficiencies using 12-, 10-, and 8-nucleotide-long ss tails.

To test this cloning procedure, human genomic DNA was amplified with Alu primer PDJ8312 (12-nucleotide ss tail), PDJ8310 (10-nucleotide ss tail), and PDJ8308 (8-nucleotide ss tail) in separate reactions. With each primer complex PCR mixtures have been obtained (Fig. 2). The size of the DNA fragments is in the range of $150-3000 \mathrm{bp}$. For the generation of the vector DNA (pUC19), $X b a I$-cut plasmid was amplified with the primer pairs PDJ8012/PDJ8112 (12-nucleotide ss tails), PDJ8010/PDJ8110 (10nucleotide ss tails), and PDJ8008/ PDJ8108 (8-nucleotide ss tails), respectively. About 2-3 $\mu \mathrm{g}$ of linear plasmid per $50-\mu \mathrm{l}$ PCR reaction was obtained starting with $1 \mathrm{ng}$ of Xbal-cut pUC19 (Fig. 2).
To correlate the cloning efficiency to the purification status of the vector and the Alu PCR products, experiments with the 12-nucleotide ss tails were performed. The Alu PCR products (PDJ8312) and the linear vector PCR products (PDJ8012/PDJ8112) were treated with T4 DNA polymerase to generate the complementary overhangs. The polymerase was heat inactivated and the PCR fragments were either further purified with GeneClean or were used without purification in LIC. Appropriate dilutions of both components were done in TE buffer. Vector (pUC19, $10 \mathrm{ng}$ ) and interAlu fragments (60 ng) were mixed in a $20-\mu 1$ volume (TE buffer) and were incubated for $5 \mathrm{~min}$ to allow the formation of recombinant molecules. One-fourth of the mixture was used for bacterial transformation without ligation. The transformed cells were plated on LB ampicillin plates. From $2.5 \mathrm{ng}$ of pUC19, 227 transformants were obtained when the vector and the insert were further purified. When both LIC partners were not purified, 163 transformants were obtained. In cases where either the insert or

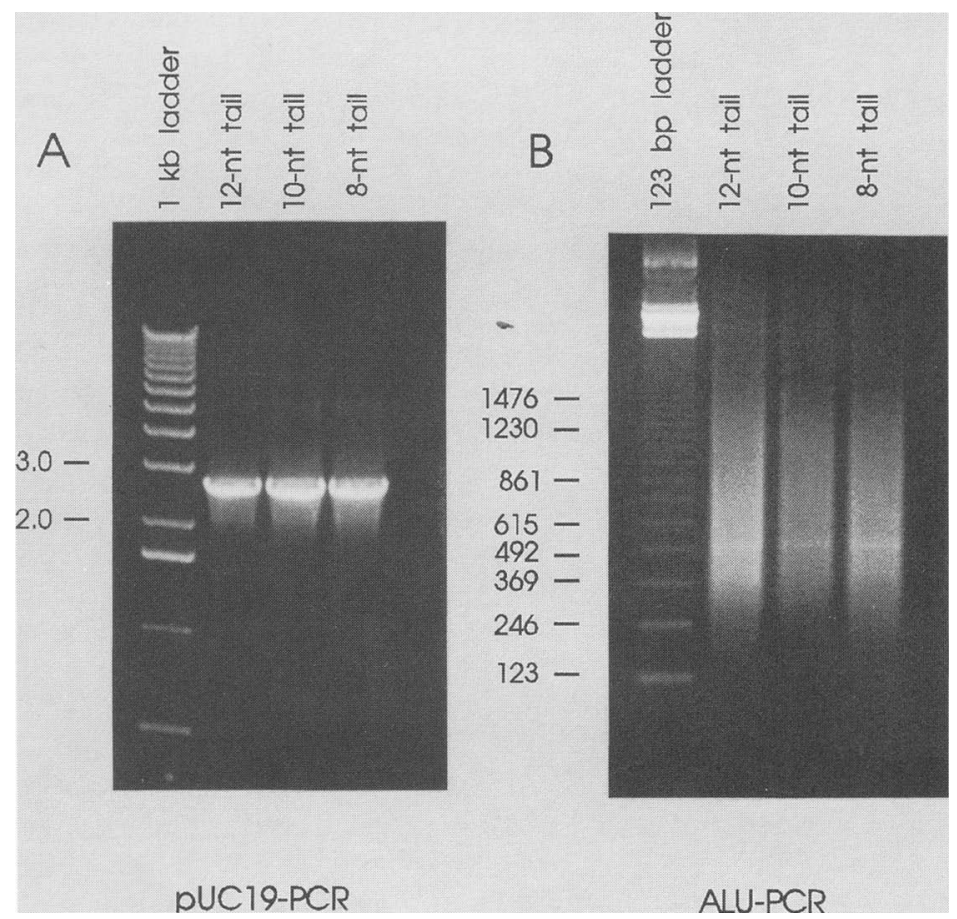

FIGURE 2 (A) pUC19 vector PCR. Xbal-cut pUC19 (1 ng) was amplified using the primer pairs PDJ8012/PDJ8112 (12-nucleotide tail), PDJ8010/PDJ8110 (10-nucleotide tail), and PDJ8008/ PDJ8108 (8-nucleotide tail). Five microliters of a 50- $\mu$ l PCR reaction was analyzed by gel electrophoresis in $0.7 \%$ agarose. The $1-\mathrm{kb}$ ladder (BRL) was used as standard (size in $\mathrm{kb}$ ). (B) Alu PCR from genomic DNA. PCR products were generated with Alu primer PDJ8312 (12-nucleotide tail), PDJ8310 (10-nucleotide tail), and PDJ8308 (8-nucleotide tail). Ten microliters of a 50- $\mu$ l PCR reaction was separated in $1.2 \%$ agarose. The 123 -bp ladder (BRL) was used as standard (size in bp).

the vector was purified, 245 or 152 transformants were obtained, respectively. These differences probably result from minor variations in the vector concentration in the individual experiments and are not caused by purification effects.

To determine the cloning efficiencies of PCR products having 10- or 8-nucleotide-long ss tails we have used Alu PCR products and vector DNA amplified with the appropriate primers in LIC. The conditions for the cloning were as given above for the experiment with nonpurified vector and purified Alu PCR products. Whereas fragments with 12-nucleotide-long overhangs resulted in 170 transformants per 2.5-ng vector, only 43 transformants were obtained when 10nucleotide overhangs were used (Table 1 ). No colonies were detected on plates when 8-nucleotide-long ss tails were used for cloning. For the uptake of DNA by the DH5 $\alpha$ cells, a heat shock for $45 \mathrm{sec}$ at $42^{\circ} \mathrm{C}$ was required. This temperature was monitored carefully because the transformation efficiency dropped dramatically at slightly higher temperatures. Because the noncovalent-associated DNA fragments with 8- and 10nucleotide overhangs may dissociate more easily at $42^{\circ} \mathrm{C}$ compared with 12 nucleotide ss tails, we lowered the temperature for the heat shock. Heating the cells for $5 \mathrm{~min}$ at $37^{\circ} \mathrm{C}$ did not improve the results. In contrast, only $25-50 \%$ of the transformants were obtained (data not shown). However, the transformation efficiency of the DH5 $\alpha$ cells was not affected, as could be seen when covalent closed circles of pUC19 were used.

To characterize the recombinants from different experiments, randomly picked transformants from several cloning experiments were analyzed for inserts by PCR with primers flanking the cloning site of the vector (universal and reverse pUC19 primers). When LIC was performed using the 12-nucleotide overhangs, 57 of 59 transformants contained inserts. As for the LIC with the 10-nucleotide ss tails, 38 of 39 transformants contained inserts. Both cloning procedures seem to result in comparable frequencies of recombinants (>96\%). Considering the fact that screening for recombinants is not used in LIC, $96 \%$ recombinant transformants reflects the high efficiency of this technique. The fraction of the recombinants can be raised theoretically to $100 \%$ if the T4 DNA polymerase- 
TABLE 1 Transformation Efficiencies Using 12-, 10-, and 8-nucleotide ss tails

\begin{tabular}{ccccc}
\hline $\begin{array}{l}\text { LIC tail } \\
\text { (nucleotides) }\end{array}$ & $\begin{array}{l}\text { Vector } \\
(2.5 \mathrm{ng})\end{array}$ & $\begin{array}{l}\text { Insert } \\
(15 \mathrm{ng})\end{array}$ & Transformants \\
\hline $3 \mathrm{a}$ & 12 & - & + & 170 \\
$3 \mathrm{~b}$ & 10 & - & + & 43 \\
$3 \mathrm{c}$ & 8 & - & + & 0 \\
\hline
\end{tabular}

The numbers of transformants using $2.5 \mathrm{ng}$ of T4 DNA polymerase-treated pUC19 vector (not purified, -) and $15 \mathrm{ng}$ of T4 DNA polymerase-treated Alu PCR products (purified, +), resulting from experiments with 12-, 10-, or 8-nucleotide-long overhangs, are given.

treated vector is further purified from agarose gels to eliminate the minute amount of pUC19 that was not cut by XbaI. ${ }^{(9)}$

To demonstrate that the recombinants were generated properly by virtue of the predetermined 12 - or 10-nucleotide ss tails, DNA from five transformants each was isolated and sequenced with pUC19-specific, fluorescence-labeled primers. All clones contained the properly ligated LIC sequences at both ends of the inserts (data not shown).

\section{DISCUSSION}

The ability of T4 DNA polymerase to degrade the $3^{\prime}$ ends of PCR fragments in a controlled fashion so that $5^{\prime}$ ss tails of predetermined DNA sequence and length are generated has been utilized to develop LIC techniques. In our original LIC protocol, we generated 12-nucleotide ss tails in the PCR fragment and in the PCR-amplified plasmid vector. ${ }^{(9)}$ The 12-nucleotide 5' extending vector ends created by our procedure permit the formation of stable duplexes with cohesive tails from the PCR products, thus eliminating the need for ligation. The cloning procedure is simple because only a single type of enzymatic reaction is required prior to transformation and results in a high cloning efficiency (up to $5 \times 10^{5}$ recombinants/ $\mu \mathrm{g}$ of vector). Nonrecombinant clones are practically eliminated.

The 12-nucleotide ss tails proved to be the minimal length for efficient LIC. When we used 10-nucleotide ss tails at the vector and insert DNA fragments, the transformation efficiency dropped to $\sim 25 \%$. With 8 -nucleotide ss tails no transformants were obtained. This decrease in transformation efficiency was not caused solely by the reduced stability of 10-nucleotide versus 12-nucleotide heteroduplexes during the heat shock of the transformation process $(45 \mathrm{sec}$ at $42^{\circ} \mathrm{C}$ ). Lowering the temperature for the heat shock to $37^{\circ} \mathrm{C}$ did not improve the transformation efficiency. Similar observations have been made also by Rashtchian et al. ${ }^{(12)}$ These investigators used the uracil DNA glycosylase to generate ss tails at the ends of vector and clonable PCR fragments. They state that products with 12-nucleotide ss tails cloned 5-10 times more efficiently than those with 9-nucleotide ss tails, and no transformants were obtained with 6-nucleotide cohesive ends. In contrast to our protocol, they used a repeated sequence for the annealing region $\left[(\mathrm{CUA})_{4}\right]$. This resulted in some heterogeneity among the recombinants produced because the annealing of ends could take place involving some or all of the CUA repeats. These workers also have emphasized the high cloning efficiency when 12 -nucleotide ss tails are used $\left(1 \times 10^{5}\right.$ to $9 \times 10^{5}$ transformants/ $\mu$ g vector).

In a similar cloning procedure (in vivo cloning, IVC) Oliner et al. ${ }^{(28)}$ clearly demonstrated that the efficiency of LIC is dependent on (1) the procedure used to purify the inserts, and (2) the length of the cohesive ends generated. Using electroporation, they obtained 100,000 clones per microgram of vector $(>90 \%$ recombinants) when both ends were 22 and 27 nucleotides long. With cohesive ends up to 67 nucleotides the transformation efficiencies were raised to $4 \times 10^{5}$ to $6 \times 10^{5}$. Knowing that electroporation is much more efficient than the transformation protocol used in our study, the transformation efficiency achieved by our procedure is very competetive. It may be that the high efficiency observed with the LIC 12 primers reflects a peculiarity of the specific DNA sequence or the protocol used in our study. Therefore, we did not consider it necessary to perform experiments with longer LIC tails. Longer tails would render the primers too long and too expensive.
The LIC procedure as described in this article is completed in $1 \mathrm{hr}$. For the T4 DNA polymerase treatment of the PCR fragments and the PCR amplified vector it is necessary to purify these products. This can easily be done in 20 min with commercially available kits based on a glass powder matrix. The subsequent polymerase treatment $(20 \mathrm{~min})$ and inactivation of the enzyme (10 $\mathrm{min})$ are followed by a 5 -min incubation of vector and insert prior to transformation of the bacteria. The incubation of vector and insert DNA is in the presence of TE buffer. No heteroduplex stabilizing agents are required. For efficient cloning the insert has to be in molar excess over the vector $(>10)$. Because of the high efficiency of this cloning procedure, as little as $10 \mathrm{ng}$ of vector is used for routine subcloning of PCR products. It should be kept in mind that one 50- $\mu$ l PCR reaction results in $2-3 \mu \mathrm{g}$ of amplified vector.

Other investigators have designed plasmid vectors that upon digestion with a specific restriction endonuclease, allow the specific degradation of the $3^{\prime}$ ends using T4 DNA polymerase to generate 12-nucleotide-long ss tails. Haun et al. $^{(20)}$ have engineered a pBluescript vector that facilitates the generation of 12 nucleotide cohesive ends upon digestion with Narl. The nonrecombinant fraction of transformants is $<15 \%$ in their experiments. These workers state that although the linearized vector was gel purified, the nonrecombinants resulted from plasmid DNA that had not been digested with NarI. The nonrecombinant fraction in our LIC is very low because the vector is amplified from small amounts of Xbal-cut pUC19 (1 ng). In theory, $<1 \mathrm{pg}$ of $\mathrm{XbaI}$-cut plasmid is contained in $10 \mathrm{ng}$ of amplified vector.

However, if functional cloning vectors are required for the cloning of cDNAs as fusion proteins, it is advisable to use nonamplified plasmid vectors to overcome possible mutations in relevant DNA sequences introduced by Taq polymerase. Haun and Moss ${ }^{(19)}$ have constructed plasmid vectors that facilitate LIC of GST fusion genes for expression in E. coli. Kuijper et al. ${ }^{(29)}$ have designed cloning vectors ("Prime" cloning vectors) that include phage $\lambda$ and plasmid vectors useful for functional cloning in oocytes, yeast, and mammalian cells, and their use in a "Prime" cloning system. They also make use of the $\left(3^{\prime} \rightarrow 5^{\prime}\right)$ 


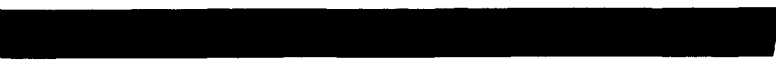

exonuclease activity of T4 DNA polymerase to generate 15- and 16-nucleotide-long ss tails for cloning. Based on our LIC ${ }^{(9)}$ and the procedure by Haun et al. ${ }^{(19,20)}$ a panel of LIC vectors for various applications, including cloning and mammalian and baculoviral expression, has been engineered by S. Gruenwald and S. Singh (PharMingen, San Diego, CA).

The results described in this report demonstrate the ease and applicability of the LIC technique to a variety of PCR products. From several independent experiments a $100 \%$ recombinant fraction of clones was achieved ( $>400$ independently picked transformants) when the amplified vector was gel purified. ${ }^{(9)}$ Here, we have shown that if the vector is not gel purified the recombination fraction of the clones is still very high $(>96 \%)$. The manipulations of the vector and the insert and the incubation times prior to cloning have been minimized. These properties make LIC a general and versatile method for cloning of PCR products.

\section{ACKNOWLEDGMENTS}

The excellent technical assistance of Ulrike Stöckl is acknowledged.

\section{REFERENCES}

1. Saiki, R.K., D.H. Gelfand, S. Stoffel, S.J. Scharf, R. Higuchi, G.T. Horn, K.B. Mullis, and H.A. Erlich. 1988. Primer-directed enzymatic amplification of DNA with a thermostable DNA polymerase. Science 239: 487-491.

2. Luedecke, H.J., G. Senger, U. Claussen, and B. Horsthemke. 1989. Cloning defined regions of the human genome by microdissection of banded chromosomes and enzymatic amplification. Nature 338: 348-350.

3. Johnson, D.H. 1990. Molecular cloning of DNA from specific chromosomal regions by microdissection and sequence-independent amplification of DNA. Genomics 6: 243-251.

4. Brooks-Wilson, A.R., P.N. Goodfellow, S. Povey, H.A. Nevanlinna, P.J. de Jong, and P.J. Goodfellow. 1990. Rapid cloning and characterization of new chromosome 10 DNA markers by Alu element-mediated PCR. Genomics 7: 614-620.

5. Cotter, F.E., G.M. Hampton, S. Nasipuri, W.F. Bodmer, and B.D. Young. 1990. Rapid isolation of human chromosomespecific DNA probes from a somatic cell hybrid. Genomics 7: 257-263.
6. Nelson, D.L., S.A. Ledbetter, L. Corbo, M.F. Victoria, R. Ramirez-Solis, T.D. Webster, D.H. Ledbetter, and C.T. Caskey. 1989. Alu polymerase chain reaction: A method for rapid isolation of human-specific sequences from complex DNA sources. Proc. Natl. Acad. Sci. 86: 66866690.

7. Clark, J.M. 1988. Novel non-templated nucleotide addition reactions catalyzed by procaryotic and eucaryotic DNA polymerases. Nucleic Acids Res. 16: 9677-9686.

8. Hemsley, A., N. Arnheim, M.D. Toney, G. Cortopassi, and D.J. Galas. 1989. A simple method for site-directed mutagenesis using the polymerase chain reaction. $\mathrm{Nu}$ cleic Acids Res. 17: 6545-6551.

9. Aslanidis, C. and P.J. de Jong. 1990. Ligation-independent cloning of PCR products (LIC-PCR). Nucleic Acids Res. 18: 6069-6074.

10. Shuldiner, A.R., L.A. Scott, and J. Roth. 1990. PCR-induced (ligase-free) subcloning: A rapid reliable method to subclone polymerase chain reaction (PCR) products. Nucleic Acids Res. 18: 1920.

11. Shuldiner, A.R., K. Tanner, L.A. Scott, C.A. Moore, and J. Roth. 1991. Ligase-free subcloning: A versatile method to subclone polymerase chain reaction (PCR) products in a single day. Anal. Biochem. 194: 9-15.

12. Rashtchian, A., G.W. Buchman, D.M. Schuster, and M.S. Berninger. 1992. Uracil DNA glycosylase-mediated cloning of polymerase chain reaction-amplified DNA: Application to genomic and cDNA cloning. Anal. Biochem. 206: 91-97.

13. Cease, K.B. and C.J. Lohff. 1993. A vector for facile PCR product cloning and modification generating any desired 4-base $5^{\prime}$ overhang:pRPM. BioTechniques 14: 250 255.

14. Dietmaier, W., S. Fabry, and R. Schmitt. 1993. DISEC- TRISEC: Di- and trinucleotide-sticky-end cloning of PCR-amplified DNA. Nucleic Acids Res. 21: 36033604.

15. Holton, T.A. and M.W. Graham. 1991. A simple and efficient method for direct cloning of PCR products using ddT-tailed vectors. Nucleic Acids Res. 19: 1156.

16. Marchuk, D., M. Drumm, A. Saulino, and F.S. Collins. 1991. Construction of T-vectors, a rapid and general system for direct cloning of unmodified PCR products. Nucleic Acids Res. 19: 1154.

17. Mead, D.A., N.K. Pey, C. Herrnstadt, R.A. Marcil, and L.A. Smith. 1991. A universal method for the direct cloning of PCR amplified nucleic acid. Biotechnology 9: 657663.

18. Hu, G. 1993. DNA polymerase-catalyzed addition of nontemplated extra nucleotides to the $3^{\prime}$ end of a DNA fragment. DNA Cell Biol. 12: 763-770.

19. Haun, R.S. and J. Moss. 1992. Ligation- independent cloning of glutathione S-transferase fusion genes for expression in Escherichia coli. Gene 112: 37-43.

20. Haun, R.S., I.M. Servent, and J. Moss. 1992. Rapid, reliable ligation-independent cloning of PCR products using modified plasmid vectors. BioTechniques 13: $515-518$.

21. Hamel, C.P., E. Tsilou, B.A. Pfeffer, J.J. Hooks, B. Detrick, and T.M. Redmont. 1993. Molecular cloning and expression of RPE65, a novel retinal pigment epithelium-specific microsomal protein that is post-transcriptionally regulated in vitro. $I$. Biol. Chem. 268: 15751-15757.

22. Serventi, I.M., E. Cavanaugh, J. Moss, and M. Vaughan. 1993. Characterization of the gene for ADP-ribosylation factor (ARF) 2, a developmentally regulated, selectively expressed member of the ARF family of $\sim 20-\mathrm{kDa}$ guanine nucleotidebinding proteins. J. Biol. Chem. 268: 4863-4872.

23. Viera, J. and J. Messing. 1987. Production of single-stranded plasmid DNA. Methods Enzymol. 153: 3-11.

24. Sambrook, J., E.F. Fritsch, and T. Maniatis. 1989. Molecular cloning: A laboratory manual. Cold Spring Harbor Laboratory, Cold Spring Harbor, New York.

25. Jelinek, W.R. and C.W. Schmid. 1982. Repetitive sequences in eucaryotic DNA and their expression. Annu. Rev. Biochem. 51: $813-844$.

26. Jurka, J. and T. Smith. 1988. A fundamental division in the Alu family of repeated sequences. Proc. Natl. Acad. Sci. 85: 47754778

27. Kariya, Y., K. Kato, Y. Hayashizaki, S. Himeno, S. Tarui, and K. Matsubara. 1987. Revision of consensus sequence of human Alu repeats. Gene 53: 1-10.

28. Oliner, J.D., K.W. Kinzler, and B. Vogelstein. 1993. In vivo cloning of $\mathrm{PCR}$ products in E. coli. Nucleic Acids Res. 21: 5192-5197.

29. Kuijper, J., K.M. Wiren, L.D. Mathies, C.L. Gray, and F.S. Hagen. 1992. Functional cloning vectors for use in directional cDNA cloning using cohesive ends produced with T4 DNA polymerase. Gene 112: $147-155$.

Received July 28, 1994; accepted in revised form September 12, 1994. 


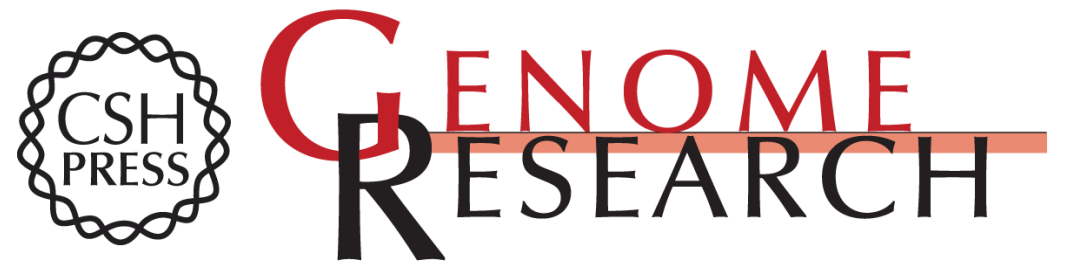

\section{Minimal length requirement of the single-stranded tails for ligation-independent cloning (LIC) of PCR products.}

C Aslanidis, P J de Jong and G Schmitz

Genome Res. 1994 4: 172-177

References This article cites 28 articles, 5 of which can be accessed free at: http://genome.cshlp.org/content/4/3/172.full.html\#ref-list-1

License

Email Alerting Receive free email alerts when new articles cite this article - sign up in the box at the Service top right corner of the article or click here.

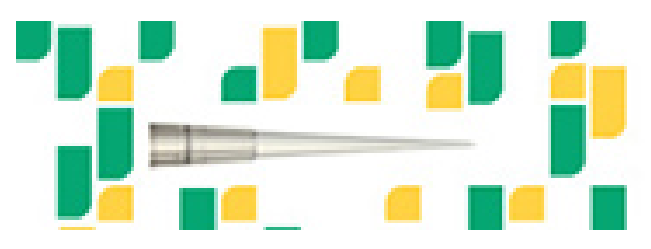

Focused on your science.

To subscribe to Genome Research go to:

https://genome.cshlp.org/subscriptions 